\title{
Treatment of Problem Alcohol Use in Women of Childbearing Age: Results of a Brief Intervention Trial
}

\author{
Linda Baier Manwell, Michael F. Fleming, Marlon P. Mundt, Ellyn A. Stauffacher, and Kristen Lawton Barry
}

\begin{abstract}
Background: Studies suggest that $14 \%$ of women age 18 to 40 drink alcohol above recommended limits Of special concern is the increasing use of alcohol by women during pregnancy. This article reports 48 month follow-up data from a subanalysis of a trial for early alcohol treatment (Project TrEAT) focused on women of childbearing age.

Methods: Project TrEAT was conducted in the offices of 64 primary care, community-based physicians from 10 Wisconsin counties. Of 5979 female patients ages 18 to 40 who were screened for problem drinking, 205 were randomized into an experimental group $(n=103)$ or control group $(n=102)$. The intervention consisted of two $15 \mathrm{~min}$, physician-delivered counseling visits that included advice, education, and contracting by using a scripted workbook. A total of 174 subjects $(85 \%)$ completed the 48 month follow-up procedures.

Results: No significant differences were found between the experimental and control groups at baseline for alcohol use, age, socioeconomic status, smoking, depression or anxiety, conduct disorder, lifetime drug use, or health care utilization. The trial found a significant treatment effect in reducing both 7 day alcohol use $(p=0.0039)$ and binge drinking episodes $(p=0.0021)$ over the 48 month follow-up period. Women in the experimental group who became pregnant during the follow-up period had the most dramatic decreases in alcohol use. A logistic regression model based on a $20 \%$ or greater reduction in drinking found an odds ratio of 1.93 (confidence interval 1.07-3.46) in the sample exposed to physician intervention. Age, smoking, depression, conduct disorder, antisocial personality disorder, and illicit drug use did not reduce drinking significantly. No significant differences were found in health care utilization and health status between groups.

Conclusions: This trial provides the first direct evidence that brief intervention is associated with sustained reductions in alcohol consumption by women of childbearing age. The results have enormous implications for the U.S. health care system.
\end{abstract}

Key Words: Alcohol, Counseling, Brief Intervention, Women, Pregnancy.

A LCOHOL USE DISORDERS are an important public health problem for women of childbearing age. Consequences of heavy alcohol use may include chronic depression, panic attacks, partner violence, unwanted sexual experiences, fetal alcohol exposure, fetal alcohol syndrome, child abuse, and numerous medical problems (National Institute on Alcohol Abuse and Alcoholism, 1997). The increasing use of alcohol by women during pregnancy is of special concern to clinicians and policymakers (Institute of Medicine, Committee of Study Fetal Alcohol Syndrome, 1996). Data from the Centers for Disease Control and Prevention Behavioral Risk Factor Surveillance System in-

From the Center for Addiction Research and Education (LBM, MFF, MPM, EAS), University of Wisconsin-Madison Medical School, Madison, Wisconsin; and the Veterans Administration Field Unit, HSR\&D, and University of Michigan Department of Psychiatry (KLB), Ann Arbor, Michigan.

Received for publication February 17, 2000; accepted July 21, 2000.

This work was supported by Grant AA 08512-01A from the NLAAA, and Grant 027204 from The Robert Wood Johnson Foundation.

Reprint requests: Linda Baier Manwell, B.S., Center for Addiction Research and Education, Department of Family Medicine, 777 S. Mills Street, Madison, WI 53715; Fax 608-263-5813; Imanwell@fammed.wisc.edu

Copyright (C) 2000 by the Research Society on Alcoholism. dicate that rates of "frequent drinking" (seven or more drinks per week or five or more drinks on any one occasion) by pregnant women increased from $0.8 \%$ in 1991 to $3.5 \%$ in 1995 (MMWR, 1997). Rates of any alcohol use during pregnancy increased from $12 \%$ to $16 \%$ during this time period. Of women of childbearing age, $4 \%$ reported consuming more than seven drinks per week, and $10 \%$ percent reported consuming five or more drinks on any one occasion. There was a great deal of variability by state. Wisconsin had the highest prevalence. More than $19 \%$ of the female population age 18 to 44 reported drinking more than seven drinks per week or five or more drinks on any one occasion (MMWR, 1997).

As a result of these data, effective alcohol abuse prevention strategies for women have become an important national research priority for public and private institutions (U.S. Department of Health and Human Services, 1993, 1997, 1998, 2000). One method of active prevention research is the use of brief intervention techniques in clinical settings to reduce alcohol use in nondependent, problem drinkers. These clinically based interventions include assessment and direct feedback, contracting and goal setting, 
behavior modification techniques (e.g., motivational interviewing and cognitive behavioral therapy), and the use of written materials such as self-help manuals (Babor, 1990; Heather et al., 1987; Miller, 1987; Miller and Baca, 1983).

A number of randomized clinical trials have tested the efficacy of brief interventions in reducing alcohol use, adverse health effects, and health care utilization in both men and women (Fleming and Manwell, 1999). The brief intervention procedures varied by trial, but most consisted of a 5 to 20 min counseling session with a variable number of follow-up sessions. Reviews conducted by Bien et al. (1993), Kahan et al. (1995), and Wilk et al. (1997) found a positive effect in the majority of studies. The meta-analysis conducted by Wilk et al. (1997) with pooled data from 12 primary care trials found a combined odds ratio of 1.9 with a confidence interval of 1.6 to 2.2 in favor of brief alcohol interventions over no intervention. None of these trials, however, focused on women of childbearing age.

Since these meta-analyses were performed, two randomized trials with pregnant women have been reported. Hankin et al. (2000) performed a trial with a large sample of pregnant women from a large metropolitan area. They found decreased alcohol use by the women assigned to the physician-delivered brief advice group and improved outcomes in their infants. In a second study of pregnant women, Chang et al. (1999) randomly assigned 250 pregnant women to an assessment-only group or to a brief intervention-assessment group. Women in their second trimester were eligible if they had a positive score on the T-ACE (Sokol et al., 1989). Although there were significant reductions in alcohol use within groups in the antenatal and postpartum period, minimal differences were found between the control and intervention groups. Marlatt and colleagues (1998) conducted a study to test the efficacy of brief counseling on reducing alcohol use and alcoholrelated problems in college students. The trial randomized 348 heavy drinking male and female students into a control or brief intervention group. Women college students in the sample had a greater reduction in alcohol problems scores compared with the men.

Here we report 48 month follow-up data from a subanalysis of a Trial for Early Alcohol Treatment (Project TrEAT) focused on women of childbearing age. Project TrEAT 12 month data initially were reported in 1997 (Fleming et al., 1997). The present report includes new information on the long-term efficacy of brief physician advice in reducing alcohol use, health status, and utilization in a previously understudied population. This is the first report in the world literature focused on women of childbearing age in primary care settings.

\section{METHODS}

\section{Physicians}

Physicians were recruited through the Wisconsin Research Network, community hospitals, managed care organizations, and personal contacts.
Physician eligibility criteria included (1) trained in family or internal medicine, (2) practicing at least $50 \%$ time, (3) based in a community primary care clinic, (4) amenable to participating in a training program, and (5) amenable to following the research protocol. Approximately $50 \%$ $(n=64)$ of the physicians who attended the $30 \mathrm{~min}$ recruitment sessions decided to participate. Most refusals involved time limitations due to an inadequate number of physicians at a given site. The 46 male and 18 female participating physicians had a mean age of 46 and an average of 13 years in practice. Their 17 practice sites varied from rural solo clinics to large urban health maintenance organization (HMO) groups. Fifty-three percent of these physicians $(n=34)$ had received training in alcohol use disorders in medical school or residency.

Physicians were trained to administer the intervention protocol through role-playing and general skills training techniques in educational programs conducted at each of the 17 clinics. The physicians also received additional training in booster sessions as subjects were randomized into the trial over a 9 month period. Physicians or their practices were paid $\$ 300$ for participating in the study.

\section{Procedures}

The research protocol and consent forms were reviewed and approved by the University of Wisconsin Committee for the Protection of Human Subjects. All patients ages 18 to 64 with regularly scheduled appointments between April 1992 and April 1994 were asked by reception personnel to complete a Health Screening Survey (HSS). Only 5979 female patients age 18 to 40 are considered in this report. The $5 \mathrm{~min}$ HSS, designed as a general lifestyle questionnaire to minimize the intervention effect of the aicohol questions, contained parallel questions on exercise, smoking, weight, and alcohol use in the last 6 months (Fleming and Barry, 1991; Wallace and Haines, 1985). Patient refusal to complete the HSS varied by clinic with a range of $2 \%$ to $30 \%$ and a weighted mean of $13 \%$ for an $87 \%$ response rate.

Problem drinkers were defined as women who drank more than 11 standard drinks per week ( $132 \mathrm{~g}$ of alcohol), consumed more than four standard drinks per occasion, or gave two or more positive responses to the CAGE questions. Figure 1 illustrates participant flow. The 730 subjects who screened positive on the HSS for problem drinking were contacted by a researcher and invited to participate in a face-to-face intervicw to determine eligibility for the trial. A total of 454 patients participated in this $30 \mathrm{~min}$ assessment interview. The interview, conducted by the researcher in each patient's primary care clinic, gathered detailed alcohol use data as well as information on licit and illicit drug use, injuries, emergency department visits, hospitalizations, health status, family function, and mental health. Two hundred and five subjects met eligibility criteria and were randomized by computer to an experimental $(n=103)$ or control $(n=102)$ group. Patients were excluded from the study if they were pregnant, had undergone alcohol treatment in the previous year $(n=10)$, reported symptoms of alcohol withdrawal in the last 12 months $(n=3)$, received physician advice to change their alcohol use in the previous 3 months $(n=52)$, consumed more than 56 drinks per week $(n=1)$, or reported suicidal thoughts $(n=14)$. Most subjects did not meet inclusion criteria because their alcohol use in the previous 7 days was below the selected cutoff limit. Other reasons for exclusion included lack of interest in participating and severe medical problems.

Subjects assigned to the control group received a booklet on general health issues and were instructed to address any health concerns in their usual manner. Patients randomized to the experimental group were given the same booklet and were scheduled to see their personal physician for the brief intervention treatment. The brief intervention protocol consisted of a workbook that contained feedback on current health behaviors, a review of the prevalence of problem drinking, a list of the adverse effects of alcohol, a worksheet on drinking cues, a drinking agreement in the form of a prescription, and drinking diary cards. The intervention was based on protocols developed for the Medical Research Council trial (Wallace et al., 1988). Two 15 min visits with the physician were scheduled 1 month apart (brief intervention and reinforcement session). Patients received a 
Health Screening Survey (HSS) Self-administered screening test given to women aged $18-40$ years entering clinic waiting rooms
Fig. 1 Sample flow.

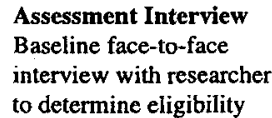

to determine eligibility
12-Month Follow-up Telephone Interview

24-Month Follow-up Telephone Interview

36-Month Follow-up Telephone Interview

48-Month Follow-up Telephone Interview supportive follow-up phone call from the clinic nurse 2 weeks after each physician visit.

Seventy of the 103 experimental subjects received the intervention protocol; of these, 28 participated in only one of the two physician visits. Thirty-three women failed to keep any appointment with their physician and did not receive the intervention. These subjects were rescheduled to receive the intervention at least three times. Primary reasons given by these patients for not participating in the scheduled intervention included childcare issues, transportation problems, and inability to take time from work. This group was not statistically different at baseline from the persons who completed the intervention on age, alcohol use, health services utilization, employment status, marital status, education, or frequency of mental illness. Of the 33 experimental subjects who did not receive the intervention, 28 participated in the follow-up interviews over the 48 months. All persons initially randomized to the experimental group $(n=103)$ remained in this group for the analysis. Intention-to-treat procedures were followed in the analysis.

All control and experimental subjects received follow-up telephone interviews at $6,12,24,36$, and 48 months from one of the researchers not assigned to the subject's clinic. One hundred seventy-four subjects completed all five follow-up interviews (85\%); 200 subjects (98\%) participated in at least one of the interviews. Of the 31 women who did not complete the final 48 month interview (15\%), 17 refused, 13 were lost to follow-up, and 1 was deceased. Fourteen of the 17 subjects who refused the interview were from the experimental group. The higher rate of refusal in this group may reflect resistance to changing their alcohol use.

Health interviews designed to corroborate subjects' self-report were obtained from 172 family members (84\%) at 12 months. Family members of subjects from both the control and experimental groups consistently reported lower levels of alcohol use than the subject reported. Medical record audits were performed at 12 and 48 months. Patients were paid up to $\$ 110$ if they completed all research procedures through the 48 month follow-up interview.

\section{Measures}

The primary outcome variables included changes in alcohol use (previous 7 day use, binge drinking, excessive drinking), health care utilization (hospital days, emergency department visits), and changes in health status measures (smoking, depression, accidents, injuries). The variables were selected a priori and based on the findings of previous trials (Kristenson et al., 1983; Wallace et al., 1988; World Health Organization Brief Intervention Study Group, 1996).

\section{Analyses}

The alcohol use outcome measures, 7 day drinking total and 30 day binge drinking episodes, were analyzed by using a repeated-measures ANOVA approach (Cochran and Cox, 1957). The data were treated as independent of the physician in the analysis because scripting of the intervention should reduce the cluster effect of individual physicians, and the sample did not show evidence of a significant physician or clinic effect. Missing data were imputed in the following manner: For any subject for whom some postintervention data were available, but one or more postintervention measures were missing, the missing data items were assigned the value of the subject's postintervention average. Data for any subject for whom all postintervention data were missing were imputed from the baseline measure for that subject. Five experimental subjects and no control subjects failed to participate in any of the postintervention follow-up interviews.

We determined the significance of difference between the experimental and control groups at each of the follow-up points by using the CONTRAST(1) option of PROC GLM in SAS, where the experimental/control variable was factored into the model after controlling for the mean change from baseline (SAS Institute, 1989b). The overall significance of the treatment effect is determined by the repeated-measures ANOVA test of hypotheses for between-subject effects. 
We determined significance for the dichotomous outcome variables of excessive drinking in the past week and any binge drinking in the past month by using the Cochran-Mantel-Haenszel test, after controlling for baseline conditions (Cochran, 1954; Mantel and Haenszel, 1959). The analysis was performed with PROC FREQ in SAS (SAS Institute, 1989a).

We used Monte Carlo-based bootstrap methods to determine variability and $p$ values for health care utilization measures because, due to the highly skewed nature of utilization data with most patients requiring few or no visits to the emergency room or hospital, standard statistical tests such as $t$ tests were inappropriate (Beran and Ducharme, 1991). A logistic regression model was estimated to examine the independent effect of treatment status on a $20 \%$ reduction in alcohol use after we controlled for age, tobacco use, depression, adult personality disorder, childhood conduct disorder, and illicit drug use.

\section{RESULTS}

\section{Patient Characteristics}

Sixty-four physicians in 17 practices participated in the study. The number of persons screened at each site ranged from 44 patients in a rural, solo practitioner's office to 820 patients from a large HMO clinic. Twenty-six percent of the patients came from nine clinics in small towns, $34 \%$ came from four clinics in medium-sized cities, and $40 \%$ came from four large clinics located in the Milwaukee area. No single clinic accounted for a majority of the patients screened.

Subjects enrolled in the study ranged from 1 in a rural clinic to 26 from a closed-panel HMO clinic in Milwaukee. Eighteen percent of the patients came from nine clinics in small towns, $26 \%$ came from four clinics in medium-sized cities, and 56\% came from four clinics located in the Milwaukee area. No single clinic accounted for a majority of the patients enrolled.

Minimal differences were found at baseline between the experimental $(n=103)$ and control group $(n=102)$ subjects on several potential confounding variables (see Table 1). The age distribution was similar for each decade. The race/ethnicity distribution of the sample is similar to the distribution for primary care patients throughout Wiscon$\sin$. The population was well educated, and more than half the sample had attended at least some college. Most women worked outside the home, and unemployment was low. Forty percent of the subjects had never been married, and $15 \%$ were divorced, widowed, or separated.

Depression, childhood conduct disorder, and adult antisocial personality disorder measures were taken from the Diagnostic Interview Schedule based on the DSM-III-R (Robins et al., 1981). Nearly $50 \%$ of the women in both groups reported a history of lifetime depression, and one quarter reported depression in the last 30 days. The prevalence of childhood conduct disorders was around $10 \%$ for both groups. More than $60 \%$ of the subjects reported tobacco use in the last 6 months. The category "used mood altering drugs in the last 6 months" included marijuana, cocaine, amphetamines, LSD, illicit narcotics, and prescription drugs such as Xanax, Librium, Valium, and opioids. The rates of self-reported marijuana and cocaine use in the
Table 1. Demographic Characteristics and Health Status by Treatment Group

\begin{tabular}{|c|c|c|}
\hline & $\begin{array}{c}\text { Intervention } \\
(n=103)\end{array}$ & $\begin{array}{c}\text { Control } \\
(n=102)\end{array}$ \\
\hline \multicolumn{3}{|l|}{ Age (years) } \\
\hline $18-21$ & $14(14)$ & $13(13)$ \\
\hline $22-25$ & $19(20)$ & $15(15)$ \\
\hline $26-30$ & $30(31)$ & $23(23)$ \\
\hline $31-40$ & $37(38)$ & $50(51)$ \\
\hline \multicolumn{3}{|l|}{ Ethnicity } \\
\hline White & $82(84)$ & $83(85)$ \\
\hline Black & $8(8)$ & $10(10)$ \\
\hline Hispanic & $2(2)$ & 0 \\
\hline Native American & $1(1)$ & $2(2)$ \\
\hline Other & $4(4)$ & $2(2)$ \\
\hline \multicolumn{3}{|l|}{ Marital status } \\
\hline Married, living with partner & $45(46)$ & $41(42)$ \\
\hline Never married & $39(40)$ & $41(42)$ \\
\hline Widowed, divorced, separated & $12(12)$ & $18(18)$ \\
\hline \multicolumn{3}{|l|}{ Education } \\
\hline High school or less & $42(43)$ & $37(38)$ \\
\hline Some college & $34(35)$ & $47(48)$ \\
\hline College degree or more & $20(20)$ & $14(14)$ \\
\hline \multicolumn{3}{|l|}{ Occupation } \\
\hline Professional & $20(20)$ & $23(23)$ \\
\hline Clerical/secretarial & $18(18)$ & $17(17)$ \\
\hline Labor/machine & $16(16)$ & $16(16)$ \\
\hline Sales/services & $16(16)$ & $12(12)$ \\
\hline Student & $13(13)$ & $10(10)$ \\
\hline Homemaker & $8(8)$ & 11 (11) \\
\hline Technical/mechanics & $5(5)$ & $2(2)$ \\
\hline Unemployed & $1(1)$ & $6(6)$ \\
\hline \multicolumn{3}{|l|}{ Mental health } \\
\hline Depression in lifetime & $48(49)$ & $51(52)$ \\
\hline Depression in last 30 days & $25(25)$ & $27(27)$ \\
\hline Childhood conduct disorder & $14(14)$ & $9(9)$ \\
\hline Antisocial personality disorder & $10(10)$ & $7(7)$ \\
\hline \multicolumn{3}{|l|}{ Health behaviors } \\
\hline Exercised in last 6 months & $75(77)$ & $71(72)$ \\
\hline Smoked in last 6 months & $67(69)$ & $63(64)$ \\
\hline Used mood altering drugs in last 6 months & $36(37)$ & $40(41)$ \\
\hline Used marijuana in last 6 months & $29(30)$ & $31(32)$ \\
\hline Used cocaine in last 6 months & $8(8)$ & $9(9)$ \\
\hline
\end{tabular}

Values are percentages (numbers).

last 6 months were higher than general population samples and may reflect higher rates in primary care clinical samples and the subjects' confidence in the confidentiality of the research procedures.

\section{Alcohol Use Outcome Measures}

The major alcohol use outcome variables were average drinks per week, binge consumption, and excessive drinking. The average drinks per week, the total number of drinks in the last 7 days, was determined by 30-day Time Line Follow-Back procedures (Sobell and Sobell, 1992). Binge drinking was defined as consuming more than four drinks per occasion. Excessive drinking was defined as more than 13 drinks per week. As shown in Table 2, both groups reported decreases in all alcohol use measures through 48 months.

Differences between the experimental and the control groups were statistically significant at only a few of the individual follow-up time points, but there was long-term evidence of a significant treatment effect. As the repeated measures test for an overall treatment effect indicates, 
Table 2. Aicohol Consumption from Baseline to 48 Months by Treatment Status

\begin{tabular}{|c|c|c|c|c|c|c|}
\hline & \multicolumn{2}{|c|}{ Treatment $(n=103)$} & \multicolumn{2}{|c|}{ Control $(n=102)$} & $F$ & $p$ \\
\hline No. drinks in past 7 days & \multicolumn{2}{|c|}{ mean $(S D)$} & \multicolumn{2}{|c|}{ mean $(S D)$} & & \\
\hline Baseline & \multicolumn{2}{|c|}{$14.08(9.22)$} & \multicolumn{2}{|c|}{$14.87(8.81)$} & 0.40 & 0.53 \\
\hline 6 months & \multicolumn{2}{|c|}{$7.50(7.39)$} & \multicolumn{2}{|c|}{$10.57(6.80)$} & 2.90 & 0.09 \\
\hline 12 months & \multicolumn{2}{|c|}{$7.36(5.97)$} & \multicolumn{2}{|c|}{$11.81(11.53)$} & 5.87 & 0.01 \\
\hline 24 months & \multicolumn{2}{|c|}{$7.03(7.35)$} & \multicolumn{2}{|c|}{$10.99(14.74)$} & 3.16 & 0.08 \\
\hline 36 months & \multicolumn{2}{|c|}{$7.14(8.00)$} & \multicolumn{2}{|c|}{$9.37(10.08)$} & 0.91 & 0.34 \\
\hline 48 months & \multicolumn{2}{|c|}{$7.48(7.63)$} & \multicolumn{2}{|c|}{$9.94(10.83)$} & 1.22 & 0.27 \\
\hline Repeated measures test for overall treatment effect & \multirow{2}{*}{\multicolumn{2}{|c|}{46.7}} & & & 8.53 & 0.0039 \\
\hline$\%$ reduction from: base to 6 months & & & \multicolumn{2}{|c|}{28.9} & & \\
\hline base to 12 months & \multicolumn{2}{|c|}{47.7} & \multicolumn{2}{|c|}{20.6} & & \\
\hline base to 24 months & 50.1 & & 26.1 & & & \\
\hline base to 36 months & 49.3 & & 37.0 & & & \\
\hline base to 48 months & 46.9 & & 33.2 & & & \\
\hline Any binge drinking past 30 days & & $(\%)$ & & (\%) & & \\
\hline Baseline & & $(93.2)$ & 93 & $(91.2)$ & 0.29 & 0.59 \\
\hline 6 months & & $(59.2)$ & 77 & (75.5) & 7.34 & 0.01 \\
\hline 12 months & 62 & $(60.2)$ & 75 & (73.5) & 4.63 & 0.03 \\
\hline 24 months & & (68.9) & 83 & $(81.4)$ & 4.72 & 0.03 \\
\hline 36 months & & $(64.1)$ & 77 & $(75.5)$ & 3.55 & 0.06 \\
\hline 48 months & & $(68.0)$ & & $(69.6)$ & 0.13 & 0.71 \\
\hline$\%$ reduction from: base to 6 months & 36.5 & & 17.2 & & & \\
\hline base to 12 months & 35.4 & & 19.4 & & & \\
\hline base to 24 months & 26.0 & & 10.8 & & & \\
\hline base to 36 months & 31.3 & & 17.2 & & & \\
\hline base to 48 months & 27.1 & & 23.7 & & & \\
\hline No. binge drinking episodes past 30 days & me & (SD) & me & In (SD) & & \\
\hline Baseline & 5.1 & $(3.70)$ & 5.4 & $(4.33)$ & 0.49 & 0.49 \\
\hline 6 months & 2.2 & $(3.02)$ & 3.5 & $(3.75)$ & 2.30 & 0.13 \\
\hline 12 months & & $(2.86)$ & & $(4,65)$ & 2.63 & 0.11 \\
\hline 24 months & & $(4.23)$ & & $(5.75)$ & 4.70 & 0.03 \\
\hline 36 months & & $(4.46)$ & & $(4.50)$ & 1.17 & 0.28 \\
\hline 48 months & & $(3.78)$ & 4.5 & (5.68) & 2.21 & 0.14 \\
\hline Repeated measures test for overall treatment effect & & & & & 9.73 & 0.0021 \\
\hline$\%$ reduction from: base to 6 months & 56.3 & & 35.5 & & & \\
\hline base to 12 months & 55.5 & & 32.8 & & & \\
\hline base to 24 months & 40.4 & & 7.1 & & & \\
\hline base to 36 months & 41.6 & & 23.9 & & & \\
\hline base to 48 months & 42.2 & & 17.9 & & & \\
\hline Excessive drinking past 7 days & & $(\%)$ & & (\%) & & \\
\hline Baseline & & $(45.6)$ & 54 & $(53.0)$ & 1.10 & 0.30 \\
\hline 6 months & & $(19.4)$ & 32 & $(31.4)$ & 2.95 & 0.09 \\
\hline 12 months & 20 & $(19.4)$ & 31 & $(30.4)$ & 2.53 & 0.11 \\
\hline 24 months & 18 & $(17.5)$ & 33 & $(32.4)$ & 5.33 & 0.02 \\
\hline 36 months & 15 & (14.6) & 33 & $(32.4)$ & 8.14 & 0.004 \\
\hline 48 months & 15 & (14.6) & 23 & $(26.5)$ & 3.75 & 0.05 \\
\hline$\%$ reduction from: base to 6 months & 57.4 & & 40.7 & & & \\
\hline base to 12 months & 57.4 & & 42.6 & & & \\
\hline base to 24 months & 61.7 & & 38.9 & & & \\
\hline base to 36 months & 68.1 & & 38.9 & & & \\
\hline base to 48 months & 68.1 & & 57.4 & & & \\
\hline
\end{tabular}

Binge drinking is defined as having more than four drinks per occasion. Excessive drinking is defined as more than 13 drinks per week.

overall 7 day alcohol use $(p=0.0039)$ and number of binge drinking episodes in the previous 30 days $(p=0.0021)$ decreased significantly in the treatment group compared with the control group.

Women in the experimental group decreased their alcohol use within 6 months and maintained the reductions over the follow-up period with very little variation. They decreased their mean alcohol intake over the 48 months by $48 \%$, reducing the amount consumed in the previous seven days from 14 to 7.5 drinks per week. The number of subjects who reported any binge drinking decreased from $93 \%$ of the sample to $68 \%$. The number of binge drinking episodes decreased from five times in the previous $\mathbf{3 0}$ days to three. The number of women who drank more than 13 drinks per week decreased from 47 to 15 .

Women in the control group also reduced their alcohol use; however, these changes were different from those seen in the experimental group. Although there was a modest decrease in use at 6 months, this reduction was less than the change made by the experimental group. The 6 month level of use was maintained at the 12 and 24 month follow-ups. Then, another modest decrease in alcohol use was noted at 36 and 48 months. Although a reduction in alcohol use by the control group is a common observation in alcohol treatment trials, the etiology is not known. The reduction may be related to the intervention effect of the follow-up 
Table 3. Health Care Utilization From Baseline to 48 Months by Treatment Status

\begin{tabular}{lccc}
\hline & $\begin{array}{c}\text { Treatment } \\
(n=103)\end{array}$ & $\begin{array}{c}\text { Control } \\
(n=102)\end{array}$ \\
\hline Total no. emergency department visits & No. visits (pts) & No. visits (pts) & $p$ \\
Baseline & $23(17)$ & $29(20)$ & 0.48 \\
6 months & $14(11)$ & $20(15)$ & 0.39 \\
12 months & $23(15)$ & $21(14)$ & 0.84 \\
24 months & $23(17)$ & $27(13)$ & 0.82 \\
36 months & $35(23)$ & $32(19)$ & 0.70 \\
48 months & $11(10)$ & $20(16)$ & 0.14 \\
Total postbaseline & $106(45)$ & $120(45)$ & 0.60 \\
Total no. hospital days & No. days (pts) & No. days (pts) & $p$ \\
Baseline & $9(5)$ & $4(3)$ & 0.32 \\
6 months & $6(2)$ & $16(5)$ & 0.26 \\
12 months & $22(7)$ & $16(4)$ & 0.65 \\
24 months & $30(10)$ & $34(8)$ & 0.52 \\
36 months & $39(15)$ & $28(11)$ & 0.84 \\
48 months & $26(7)$ & $53(10)$ & 0.27 \\
Total postbaseline & $123(31)$ & $147(30)$ & 0.57 \\
\hline
\end{tabular}

pts, patients.

procedures, the regression to the mean phenomenon, or natural history changes in alcohol use in community samples.

Forty-one women in the sample experienced one or more pregnancies during the 48 -month follow-up period. The 22 women in the experimental group who became pregnant decreased their alcohol use from 13.6 to 3.5 drinks per week and from 5.7 to 1.5 binge drinking episodes per month. This reduction in use was sustained over the 48month follow-up period. The reduction was statistically different from changes in alcohol use made by the 19 women in the control group who became pregnant. Alcohol use in this group changed from 13.5 to 10.1 drinks per week and 5.5 to 4.2 binge drinking episodes per month. The combination of brief intervention and pregnancy appears to have a powerful sustained effect on alcohol use in young women.

\section{Utilization}

Table 3 illustrates the number of self-reported emergency room visits and hospital days by the experimental and control groups during the 48 months after the intervention. The experimental group reported fewer total emergency department visits-106 vs. 120 visits. The number of days that patients reported being hospitalized followed a similar pattern, with the experimental group reporting 24 fewer hospital days at the end of the 4 year period. These differences were not statistically significant, however, and may reflect an infrequent use of the hospital and emergency department by this sample. Most of the hospital days were related to admissions for pregnancy; 41 women in the sample became pregnant during the 48 month follow-up period. This absence of a health care utilization treatment effect may be related to the observation that this is a healthy population of young women with few medical problems.
Table 4. Logistic Regression Model of $20 \%$ or More Reduction in Drinking from Baseline to 48 Months

\begin{tabular}{lcc}
\hline \multicolumn{1}{c}{ Characteristic } & Adjusted odds ratio & 95\% Confidence interval \\
\hline Age & 1.18 & $0.89-1.57$ \\
Smoking in last 6 months & 1.54 & $0.81-2.92$ \\
Depressed in last 30 days & 1.50 & $0.76-2.95$ \\
Adult antisocial personality & 0.88 & $0.30-2.63$ \\
Childhood conduct disorder & 0.75 & $0.28-2.00$ \\
Illicit drug use & 1.03 & $0.56-1.91$ \\
intervention & $1.93^{*}$ & $1.07-3.46$ \\
\hline
\end{tabular}

$70 \%(n=72)$ of the experimental subjects and $54 \%(n=55)$ of the control subjects demonstrated a $20 \%$ or greater reduction in alcohol use.

${ }^{\star} p<0.03$.

\section{Health Status Measures}

No significant reductions in other outcome variables were found between groups over the 48 month follow-up period, which included the general health rating, mean number of cigarettes smoked, or number of depressive symptoms. The total number of accidents and injuries reported over the 48 months for the experimental group ( $n=$ $90)$ and the control group $(n=105)$ was also nonsignificant $(p<0.42)$.

\section{Analysis of Potential Covariates}

Table 4 presents the results of a logistic regression model that further supports the patterns observed in Table 2. The results of the model show that other demographic characteristics and health behaviors do not account for a $20 \%$ or greater reduction in alcohol consumption between the groups. The only significant predictor was exposure to the physician intervention. Age, smoking, depression, childhood conduct disorder, adult antisocial personality disorder, and illicit drug use did not significantly reduce drinking. The odds ratio $1.93(1.07-3.46)$ shown in the first column of numbers indicates that individuals in the experimental group were nearly twice as likely to reduce their drinking by $20 \%$ or more. In contrast, the odds ratio for all of the other variables is not significantly different from 1.0 , which indicates that they did not make individuals any more (or less) likely to reduce their alcohol consumption by $20 \%$.

\section{DISCUSSION}

Previously published trials conducted by Wallace et al. (1988), the World Health Organization Brief Intervention Study Group (1996), Nilssen (1991), and Israel et al. (1996) included small numbers of women of childbearing age. Project TrEAT is the first brief intervention trial to include a large sample of women in the 18 to 40 age group. The Project TrEAT subsample analysis provides compelling evidence that physician-delivered brief counseling is associated with a sustained reduction in alcohol use in these women. A clinician with 1000 female patients in his or her practice can expect that 100 women drink above recommended levels.

Based on our analysis, the clinician can expect a $20 \%$ to 
$25 \%$ reduction in average weekly consumption and episodes of binge drinking after brief intervention with these women. The trial also observed a $68 \%$ reduction $(n=47$ to $n=15$ ) in the number of women who consumed more than 13 drinks per week. The absence of statistically significant differences in health status measures and utilization events may reflect the observation that this is a healthy population with limited medical problems.

This trial's methodological strengths include a high physician retention rate and a patient follow-up rate of $85 \%$ at 48 months. This study has the longest follow-up period of all alcohol trials that included women. Corroborative family member interviews suggest that patient self-reports did not underestimate alcohol use. Intention-to-treat procedures were used, and all patients initially randomized were included in the analysis, even those who did not receive all or part of the intervention protocol. The trial had partial or complete data on $98 \%$ of the sample $(n=200 / 205)$. The trial was able to assess alcohol use, health care utilization, and health status over six time points: baseline and 6,12 , 24,36 , and 48 months.

The participation of a diverse sample of communitybased primary care practices is another strength that increases the generalizability of the findings. The clinical settings ranged from small rural solo practices to large urban multispecialty groups. Most of the physicians were part of managed care organizations. The practices were community-based rather than academic medical center teaching practices. This is important, because a majority of the health care in the United States is delivered in community-based practices. The trial screened a large sample of non-treatment-seeking high-risk female drinkers of reproductive age as opposed to subjects who were recruited by newspaper ads or referral. As a result of using community-based physicians, who traditionally provide more than $90 \%$ of the care in the United States, the findings may be applicable to primary care practices throughout the country.

Several methodological issues should be considered when interpreting the results. First, one of the primary outcome measures relied on self-report of alcohol consumption. Research indicates that self-reported alcohol consumption is more reliable than other methods of inquiry or testing (Babor et al., 1987; Maisto et al., 1990; Midanik, 1988). Methods employed in this trial to minimize selfreport bias included (1) informing patients that researchers administering the follow-up interviews were from the University of Wisconsin and not from their physician's office; (2) reassuring subjects that the information provided was confidential; (3) using follow-up surveys that contained parallel questions on weight, exercise, sleep, alcohol use, and smoking to lessen the impact of the alcohol questions; and (4) using multiple measures of alcohol use.

Another methodological issue is the concern that women who received the physician intervention may have wanted to "please the doctor" by subsequently reporting lower rates of alcohol use. In the absence of the use of a biological measure to assess recent alcohol use, family member interviews were conducted at 12 months with $84 \%$ of the sample to corroborate subject self-report. The family member interviews did not reveal any systematic difference from subject self-report by group status.

The generalizability of the results is limited to the population included in the study and may not apply to women treated for an alcohol use disorder in the past year, those with suicidal ideation, or those who drink on average more than 56 drinks per week. The trial specifically excluded alcoholics, because current standards of care require more intensive, specialized treatment for persons who are alcohol dependent. The effectiveness of the brief intervention technique also may differ for minority populations. Although the race/ethnicity of the sample matched the population of Wisconsin and the Midwest, the total number of Black, Asian, and Hispanic women precluded any subanalysis of differences by race. Intervention protocols for specific clinical sites may need to be adapted according to cultural beliefs and expectations.

\section{Summary Statement}

This trial indicates that brief advice protocols can provide a successful strategy for changing drinking behavior and improving health outcomes for high-risk female drinkers in primary care settings. Women who become pregnant may be particularly responsive to brief intervention therapy. Because $80 \%$ of U.S. women visit their physicians at least once every 2 years, brief physician advice could have enormous implications for the U.S. health care system. This trial supports the implementation of screening, assessment, and brief intervention activities for all female patients age 18 to 40 who seek health care services in community-based primary care settings.

\section{ACKNOWLEDGMENTS}

We thank John Beasley, M.D., Director of the Wisconsin Research Network; Michael French, Ph.D., Health Economist at the University of Miami; and the 64 primary care physicians who participated in the trial.

\section{REFERENCES}

Babor TF (1990) Brief intervention strategies for harmful drinkers: New directions for medical education. Can Med Assoc J 143:1070-1076.

Babor TF, Stephens RS, Marlatt GA (1987) Verbal report methods in clinical research on alcoholism: Response bias and its minimization. $J$ Stud Alcohol 48:410-424.

Beran R, Ducharme GR (1991) Asymptotic Theory for Bootstrap Methods in Statistics. CRM Publications, Montreal, PQ.

Bien TH, Miller WR, Tonigan JS (1993) Brief interventions for alcohol problems: A review. Addiction 88:315-335.

Chang G, Wilkins-Haug L, Berman S, Goetz M (1999) Brief intervention for alcohol use in pregnancy: Results from a randomized trial. Addiction 94:1499-1508.

Cochran WG (1954) Some methods for strengthening the common chi square tests. Biometrics 10:417-451. 
Cochran WG, Cox GM (1957) Experimental Design. 2nd ed. Wiley, New York.

Fleming M, Barry K (1991) A three-sample test of a masked alcohol screening questionnaire. Alcohol Alcohol 26:81-91.

Fleming MF, Barry KL, Manwell LB, Johnson K, London R (1997) Brief physician advice for problem alcohol drinkers: A randomized controlled trial in community based primary care practices. JAMA 277:1039-1045.

Fleming MF, Manwell LB (1999) Brief intervention in primary care settings: A primary treatment method for at-risk, problem, and dependent drinkers. Alcohol Res Health 23:128-137.

Hankin J, Sokol R, Shernoff N, Canestrelli J (2000) Protecting the next pregnancy. I: Impact on drinking during subsequent pregnancy (abstract). Alcohol Clin Exp Res (Suppl 5) 24:103a..

Heather N, Campion PD, Neville RG, MacCabe D (1987) Evaluation of a controlled drinking minimal intervention for problem drinkers in general practice (the DRAMS scheme). J R Coll Gen Pract 37:358-363.

Institute of Medicine, Committee of Study Fetal Alcohol Syndrome. (1996) Fetal Alcohol Syndrome: Diagnosis, Epidemiology, Prevention, and Treatment (Stratton K, Howe, C, Battaglia FC, eds.). National Academy Press, Washington, DC.

Israel Y, Hollander O, Sanchez-Craig M, Booker S, Miller V, Gingrich R, Rankin JG (1996) Screening for problem drinking and counseling by the primary care physician-nurse team. Alcohol Clin Exp Res 20:1443-1450.

Kahan M, Wilson L, Becker L (1995) Effectiveness of physician-based interventions with problem drinkers: A review. Can Med Assoc J 152: 851-859.

Kristenson H, Ohlin H, Hulten-Nosslin M, Hood B (1983) Identification and intervention of heavy drinking in middle aged men: Results and follow-up of 24-60 months of long-term study with randomized controls. Alcohol Clin Exp Res 7:203-209.

Maisto SA, McKay JR, Connors JJ (1990) Self-report issues in substance abuse: State of the art and future directions. Behav Assess 12:117-134

Mantel N, Hacnszel W (1959) Statistical aspects of the analysis of data from retrospective studies of disease. J Natl Cancer Inst 22:719-748.

Marlatt GA, Baer JS, Kivlahan DR, Dimeff DR, Larimer ME, Quigley LA, Somers JM, Williams E (1998) Screening and brief intervention for high-risk college student drinkers: Results from a 2-year follow-up assessment. J Consult Clin Psychol 66:604-615.

Midanik LT (1988) Validity of self-reported alcohol use: A literature review and assessment. Br J Addict 83:1019-1030.

Miller WR (1987) Techniques to modify hazardous drinking patterns. Recent Dev Alcohol 5:425-438.

Miller WR, Baca LM (1983) Two-year follow-up of bibliotherapy and therapist-directed controlled drinking training for problem drinkers. Behav Ther 14:441-448.

MMWR (1997) Alcohol consumption among pregnant and childbearingaged women-United States, 1991 and 1995. MMWR Morb Mortal Wkly Rep 46:346-350.
National Institute on Alcohol Abuse and Alcoholism. (1997) Epidemiology of alcohol use and alcohol-related consequences, in Ninth Special Report to the U.S. Congress on Alcohol and Health From the Secretary of Health and Human Services, September 1997 (NIH publication no. 974017), pp. 1-31. U.S. Department of Health and Human Services, Public Health Service, National Institutes of Health, National Institute on Alcohol Abuse and Alcoholism, Bethesda, MD.

Nilssen O (1991) The Tramso Study: Identification of and a controlled intervention on a population of early-stage risk drinkers. Prevent Med 20:518-528.

Robins LN, Helzer JE, Croughan JL, Ratcliff KS (1981) National Institute of Mental Health Diagnostic Interview Schedule: Its history, characteristics, and validity. Arch Gen Psychiatry 38:381-389.

SAS Institute Inc. (1989a) SAS/STAT User's Guide. vol 1, 4th ed. SAS Institute, Cary, NC.

SAS Institute Inc. (1989b) SAS/STAT User's Guide. vol 2, 4th ed. SAS Institute, Cary, NC.

Sobell LC, Sobell MB (1992) Timeline follow-back: A technique for assessing self-reported alcohol consumption, in Measuring Alcohol Consumption (Litten R, Allen J, eds), pp 41-72. Humana Press, Totowa, NJ.

Sokol RJ, Martier SS, Ager JW (1989) The T-ACE questions: Practical prenatal detection of risk-drinking. Am J Obstet Gynecol 160:863-868.

U.S. Department of Health and Human Services. (1993) Biomedical and Behavioral Research on Alcohol and Women (RFA: AA-94-001). National Institute on Alcohol Abuse and Alcoholism, Bethesda, MD.

U.S. Department of Health and Human Services. (1997) Announcement 746: Preventing Alcohol-Exposed Pregnancies Among High-Risk Women in Special Settings. Centers for Disease Control and Prevention, Atlanta, GA.

U.S. Department of Health and Human Services. (1998) Preventing Fetal Alcohol Syndrome (FAS) (RFA: AA-99-002). National Institute on Alcohol Abuse and Alcoholism, Bethesda, MD.

U.S. Department of Health and Human Services. (2000) Fetal Alcohol Syndrome (FAS) and Alcohol-Related Birth Defects. National Institute on Alcohol Abuse and Alcoholism, Small Business Technology Transfer, and Small Business Innovation Research, Bethesda, MD.

Wallace P, Cutler S, Haines A (1988) Randomized controlled trial of general practitioner intervention in patients with excessive alcohol consumption. BMJ 297:663-668.

Wallace P, Haines A (1985) The use of a questionnaire in general practice to increase the recognition of patients with excessive alcohol consumption. BMJ 290:1949-1953.

Wilk AI, Jensen NM, Havighurst TC (1997) Meta-analysis of randomized control trials addressing brief interventions in heavy alcohol drinkers. J Gen Intern Med 12:274-283.

World Health Organization Brief Intervention Study Group (1996) A cross-national trial of brief interventions with heavy drinkers. Am J Public Health 86:948-955. 